\title{
(4) The Red Cell Fragmentation Syndrome
}

\author{
Mitsuhiro Omine, MD \\ The Third Department of Internal Medicine, Gunma University School of Medicine, \\ Maebashi, Gunma 371
}

The significance of red cell fragmentation (RCF) in the general clinical practice was assessed by examining blood films for the incidence of fragmented red cells or schistocytes in approximately 1,600 patients. The schistocytes were defined as the characteristic bizarre poikilocytes observed in the typical cases with either microangiopathic hemolytic anemia or cardiac traumatic hemolytic anemia. The triangular, crescent, helmet, burr-shaped, irregularly distorted forms, microspherocytes and red cell ghosts constituted the prototypes. The incidence of schistocytes was obtained by enumerating at least 5,000 red cells. Eighty-five healthy adults showed a maximum of 1.2 schistocytes out of 1,000 red cells with the average of $0.4 \%$ according to the criteria set as above. On the other hand, in the unselected population of 661 patients (200 in- and 461 out-patients), in whom blood films had been ordered and registered in the central clinical laboratory, the overall incidence showing significantly increased schistocytes $(>3 \%$ ) was $3 \%$. The incidence was $6.5 \%$ for the hospitalized patients (mainly medical field) and $1.5 \%$ for outpatients. However the schistocyte frequency did not exceed $10 \%$ in all these cases, and the diagnosis of RGF syndrome was not feasible. Thus, the examination of a random patient population suggested that RCF is, in fact, relatively uncommon in the general clinical setting.

In order to investigate further, an unselected sequential series of patients with several disease categories was examined without prior knowledge on clinical pictures. For 176 patients with various cancer either at the time of diagnosis or hospital admission, the overall schistocyte incidence of $>3 \%$ was $14.2 \%$. Patients treated in the surgical wards showed 9 of $103(8.7 \%)$ incidence, whereas it was 10 of $73(21.9 \%)$ in the medical wards including radiology. Four of the latter had been diagnosed as RCF syndrome with schistocyte ranging $10-15 \%$. The gastric cancer, hepatoma and breast cancer were frequently associated with increased schistocytes particularly at the advanced stage of the disease.

Among 48 patients with heart diseases who were managed mostly at the out-patient-clinic, only 2, one each with valve replacement and operated VSD, showed moderate increase of schistocytes (4 and $7 \% 0$ ). The fact that the great majority of these patients had well-compensated mitral valve disease may account for the nearly normal schistocyte values. Of 55 patients with collagen-vascular diseases including Behçet's disease and Wegener's granulomatosis, 7 showed increased schistocytes. It was noted that one patient with SLE and 2 with polyarteritis nodosa were associated with significant RCF with 10 $20 \%$ of schistocytes.

Certain difficulties were encountered in patients with hematological diseases, where the defects may be intrinsic to the red cell production or destruction. In the conditions affecting predominantly the erythroid series which included megaloblastic anemia, iron deficiency anemia, sideroblastic anemia and hemopoietic dysplasia, the poikilocytes morphologically indistinguishable from typical schistocytes were increased in $18.4 \%$ of cases. The value for the conditions primarily affecting the leukocyte series; acute and chronic leukemias, malignant lymphomas and myelofibrosis was 16 of $132(12.1 \%)$. The acute leukemias and lymphomas in remission were generally not associated with red cell alterations. It appeared that a smooth contour with central pallor as well as the other morphological aberrations more or less characteristic to the individual disorders, would be a useful parameter in distinguishing the hematological disorders and RCF syndrome. The increased schistocytes were found in 12 of 30 patients $(40 \%)$ with disseminated intravascular coagulation (DIC) at the time of diagnosis. However, schistocytosis was generally mild in degree, except in some cases due to disseminated cancer. 
It may deserve a particular stress that patients treated in the intensive care unit frequently $(35 \%)$ showed a significant schistocytosis (up to $18 \% 0$ ), while some cases demonstrated an increasing trend during observation period.

Eleven patients with the unequivocal RCF syndrome were examined. It consisted of 6 thrombotic thrombocytopenic purpura, 2 cardiac traumatic, 2 metastatic cancer and 1 postoperative cancer. The schistocytes at diagnosis ranged from 11 to 164 $\%$ with a mean of $65 \%$. Meanwhile, 27 cases were listed from this survey study. Eight of these cases showed schistocytosis exceeding $10 \%$, thus suggesting the possible occurrence of a clinically silent but probably significant RCF mechanism under certain clinical conditions.

Finally the relationships of RCF with metastatic cancer were examined in patients with gastric cancer. Fifteen patients had the biopsyor autopsy-proven bone marrow metastasis (group 1), 6 showed leukoerythroblastic blood picture without positive evidence for the marrow involve- ment (group 2), and 23 cases were associated with neither one of these findings (group 3). The schistocyte incidence was $85.6 \pm 19.2,49.6 \pm$ 34.9 , and $8.9 \pm 2.2 \%$ for groups 1,2 and 3 , respectively. The severity of anemia, reticulocytosis and thrombocytopenia was most marked in group 1 and mildest in group 3. The serum LDH correlated significantly with schistocytes $(p<0.01)$. However the absence of marrow metastasis did not preclude RGF, signifying the localized cancer may give rise to a clinically significant RGF under limited circumstances.

In summary, this study has shown that the RGF syndrome is relatively uncommon in the general clinical practice. Nonetheless, if one considers the subclinical or latent RGF, then the incidence may become much more higher than that generally presumed, particularly in patients with advanced cancer, untreated collagenvascular disorders, DIC, etc. Special attention should also be paid to patients placed under the intensive care programs.

\title{
(5) Hematologic Abnormalities in Liver Disease
}

\author{
Tatsuya Ōhashi, MD and Kenji Fujiwara, MD \\ The First Department of Internal Medicine, Faculty of Medicine, \\ University of Tokyo, Tokyo
}

In order to clarify the mechanism of hematologic abnormalities in liver disease, we studied the changes in peripheral blood cell counts and hematopoiesis in association with liver function tests, splenectomy, and several blood cell changes in patients with various liver diseases.

\section{MATERIALS AND METHODS}

Patients studied were 90 with acute hepatitis, 97 with chronic hepatitis, 167 with liver cirrhosis of viral origin, 25 with alcoholic liver injury (12 with fatty liver and 13 with liver cirrhosis), 17 with fatty liver in obesity, 23 with fulminant hepatitis and 33 with idiopathic portal hypertension. The diagnosis was made histologically in all patients except for some with acute hepatitis. Blood cell counts and liver function tests were performed at the time of diagnosis. In additional 49 patients with liver cirrhosis who underwent splenectomy, blood cell counts were examined in relation to excised spleen weight. Normal subjects were 16 healthy males and 16 healthy females.

Bone marrow study was performed at the sternum. The erythropoietin activity in serum and $\beta$-thromboglobulin ( $\beta$-TG) were determined using a hemagglutination inhibition assay kit (JCL Clinical Research Corporation, Knoxville, USA) and a $\beta$-TG RIA kit (Amersham International Limited, Amersham, England), respectively. Platelet survival time ( $\mathrm{T} 1 / 2$ ) was determined by the method of Stuart et al. ${ }^{1)}$ Platelet volume was measured with a particle size distribution analyzer (Toa Medical Electronics K.K., Kobe, Japan). 